\title{
Transcatheter mitral valve repair and replacement. Expert consensus statement of the Polish Cardiac Society and the Polish Society of Cardiothoracic Surgeons
}

\author{
Wojciech Wojakowski*, Zbigniew Chmielak²*, Kazimierz Widenka³, Jerzy Pręgowski², Bartłomiej Perek4,

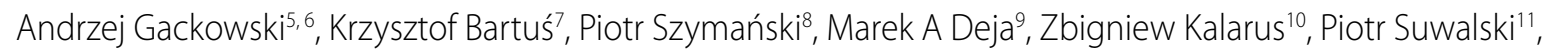 \\ Jarosław Trębacz ${ }^{12}$, Piotr Kołsut ${ }^{13}$, Piotr Ścisło ${ }^{14}$, Krzysztof Wróbel ${ }^{15}$, Grzegorz Smolka', Witold Gerber ${ }^{16}$, \\ Dariusz Dudek ${ }^{12,17}$, Tomasz Hirnle ${ }^{18}$, Marek Grygier ${ }^{19}$, Stanisław Bartuś20, Adam Witkowski2*, Mariusz Kuśmierczyk ${ }^{13 *}$ \\ Reviewers: Rafał Pawlaczyk²1, Mariusz Gąsior22, Jarosław Drożdż²3
}

\begin{abstract}
'Division of Cardiology and Structural Heart Diseases, Medical University of Silesia, Katowice, Poland 2Department of Interventional Cardiology and Angiology, National Institute of Cardiology, Warszawa, Poland ${ }^{3}$ Clinical Department of Cardiac Surgery, District Hospital No. 2, University of Rzeszow, Rzeszów, Poland ${ }^{4}$ Department of Cardiac Surgery and Transplantology, Poznan University of Medical Sciences, Poznań, Poland ${ }^{5}$ Noninvasive Cardiovascular Laboratory, John Paul II Hospital, Kraków, Poland ${ }^{7}$ Department of Cardiovascular Surgery and Transplantation, Jagiellonian University Medical College, John Paul II Hospital, Kraków, Poland ${ }^{9}$ Department of Cardiac Surgery, School of Medicine in Katowice, Medical University of Silesia, Katowice, Poland ${ }^{12}$ Institute of Cardiology, Jagiellonian University Medical College, Kraków, Poland

${ }^{13}$ Department of Cardiac Surgery and Transplantology, National Institute of Cardiology, Warszawa, Poland

${ }^{14} 11^{\text {st }}$ Chair and Department of Cardiology, Medical University of Warsaw, Warszawa, Poland

${ }^{15}$ Medicover Hospital, Warszawa, Poland

${ }^{16}$ Department of Cardiac Surgery, American Heart of Poland, Bielsko-Biała, Poland

${ }^{17}$ Maria Cecilia Hospital, GVM Care and Research, Contignola (RA), Ravenna, Italy

${ }^{18}$ Department of Cardiosurgery, University Hospital, Białystok, Poland

${ }^{19} 1^{\text {st }}$ Department of Cardiology, Poznan University of Medical Sciences, Poznań, Poland

${ }^{20} 2^{\text {nd }}$ Department of Cardiology, Institute of Cardiology, Jagiellonian University Medical College, Kraków, Poland

${ }^{21}$ Chair and Clinic of Cardiosurgery and Vascular Surgery, Medical University of Gdansk, Gdańsk, Poland

${ }^{22} 3^{\text {rd }}$ Chair and Clinical Division of Cardiology, Medical University of Silesia in Katowice, Katowice, Poland

${ }^{23} 2^{\text {nd }}$ Chair of Cardiology, Medical University of Lodz, Łódź, Poland

*These authors contributed equally to this paper
\end{abstract}

${ }^{6}$ Department of Coronary Artery Disease and Heart Failure, Institute of Cardiology, Jagiellonian University Medical College, John Paul II Hospital, Kraków, Poland

${ }^{8}$ Clinical Cardiology Center, Central Clinical Hospital of the Ministry of the Interior in Warsaw and Center of Postgraduate Medical Education, Warszawa, Poland

${ }^{10}$ Department of Cardiology, Congenital Heart Diseases and Electrotherapy, Medical University of Silesia, Silesian Centre for Heart Diseases, Zabrze, Poland ${ }^{11}$ Clinical Department of Cardiac Surgery, Central Clinical Hospital of the Ministry of Interior and Administration, Centre of Postgraduate Medical Education, Warszawa, Poland

\section{Correspondence to:}

Prof. Wojciech Wojakowski, $\mathrm{MD}, \mathrm{PhD}$,

Division of Cardiology and Structural Heart Diseases, Medical University of Silesia Ziołowa 45

40-635 Katowice, Poland, phone: +48604 188669 , e-mail:

wwojakowski@sum.edu.pl

Copyright by the Author(s), 2021

Kardiol Pol. 2021; 79 (10): 1165-1177

DOl: 10.33963/KP.a2021.0116

Received:

September 13, 2021

Revision accepted:

September 17, 2021

Published online:

September 21, 2021

\section{INTRODUCTION}

The need for an expert consensus statement of the Polish Cardiac Society and the Polish Society of Cardiothoracic Surgeons on transcatheter mitral valve repair and replacement in patients with significant mitral valve regurgitation (MR) has been triggered by dynamic progress in the development of new technologies, publication of data from randomized clinical trials and registries, increasing availability of these techniques, and growing experience of Polish operators. While in the case of patients with primary MR and acceptable operative risk, cardiac surgery is the treatment of choice (the primary surgical repair is associated with a better prognosis than valve replacement), the indications and long-term results of surgical treatment in patients with secondary (functional) MR related to left ventricular dysfunction are less documented, except for patients undergoing simultaneous revascularization or replacement of the aortic valve [1]. Patients with heart failure (HF) and secondary MR have an increased risk of mortality, a higher rate of hospitalizations for decompensated $\mathrm{HF}$, and deterioration in the quality of life. The necessity to develop alternative treatment options for patients with increased or prohibitive surgical risks with both primary and secondary MR led to rapid advances in transcatheter mitral valve repair (TMVR). The current document primarily 
discusses edge-to-edge TMVR techniques, which have been validated in randomized clinical trials and large registries in populations of patients with chronic primary and secondary MR. Other techniques of TMVR and transcatheter mitral valve implantation (TMVI) are at the early stages of clinical testing and are currently not available in Poland, except for ongoing clinical trials.

\section{TRANSCATHETER METHODS OF MITRAL VALVE REPAIR}

Transcatheter mitral valve interventions can be divided into:

1. Repair methods using CE-marked devices

a) Percutaneous repair by edge-to-edge approximation of anterior and posterior mitral valve leaflets (MitraClip and PASCAL devices), which mimics the surgical method developed by Alfieri;

b) Percutaneous reduction of mitral annulus dilatation (annuloplasty):

- Indirect (the device is implanted in the coronary sinus [Carillon]);

- Direct annuloplasty (Cardioband device);

c) Transapical implantation of artificial tendinous chords (Neochord, Harpoon);

d) Combination of the techniques listed in subparagraphs a-b.

2. Techniques of transcatheter mitral valve implantation (TMVI)

a) Implantation of balloon-expandable transcatheter aortic valve implantation (TAVI) bioprosthesis into a malfunctioning surgically implanted biological prosthesis (valve-in-valve) (CE mark for Sapien valve), surgically implanted mitral ring (valve-inring), or mitral annular calcification (valve-in-MAC);

b) Implantation of dedicated TMVI device either via transseptal (still under clinical investigation) or transapical access (currently one bioprosthesis with CE mark).

Two TMVR (percutaneous edge-to-edge repair) systems are available in Poland: MitraClip (Abbott Vascular, Santa Clara, CA, USA) and recently introduced Pascal (Edwards Lifesciences, Irvine, CA, USA). It should be emphasized that most of the data from randomized clinical trials, providing results based on the evidence-based medicine methodology and clinical experience (over 150000 patients), relate primarily to the MitraClip edge-to-edge repair system.

\section{THE EDGE-TO-EDGE REPAIR: MITRA CLIP SYSTEM}

\section{Clinical trial results}

\section{Primary (degenerative) mitral regurgitation}

Most of the currently available recommendations of scientific societies are based on the EVEREST study (Endovascular Valve Edge-to-Edge REpair STudy) and EVEREST
II utilizing the MitraClip system. In the EVEREST study, in patients disqualified from conventional surgery, the use of this system was safe and technically feasible. MR grade reduction was less effective than surgical repair/replacement due to the higher prevalence of residual MR. Nevertheless, it reduced the severity of MR (to $\leq 2+$ in approximately $75 \%$ of patients), clinical symptoms, and left ventricular remodeling [2]. The EVEREST II study involving 279 patients was the only randomized trial comparing the transcatheter approach to conventional surgery in patients with mostly primary MR. In the intention-to-treat analysis, the transcatheter procedure was less effective in MR reduction and in terms of the composite endpoint (death, surgery for mitral valve dysfunction, and grade $3+/ 4+$ MR at 2 years). This was mainly because $20 \%$ of the patients treated with MitraClip required reintervention within one year of the procedure. However, there were no differences in the mortality rates. Percutaneous treatment emerged as superior in terms of safety at 30 days, which was driven by a decreased transfusions rate. At the same time, 37 patients (20\%) in the percutaneous-repair group subsequently required mitral-valve surgery in the early postoperative period $[3,4]$. It is important to remember that in EVEREST II, 73\% of patients had primary MR and a relatively low operational risk. At the same time, modern European registries include patients with secondary MR and high or prohibitive operative risk. There is currently an ongoing randomized controlled trial to compare the clinical outcomes of the MitraClip system versus surgical repair in patients with primary mitral insufficiency who are at moderate surgical risk (REPAIR MR, NCT04198870).

\section{Secondary (functional) mitral regurgitation}

\section{Randomized trials}

The data concerning procedural success rate and effectiveness of MitraClip comes from two randomized clinical trials: MITRA-FR (Percutaneous Repair with the MitraClip Device for Severe Functional/Secondary Mitral Regurgitation) and COAPT (Cardiovascular Outcomes Assessment of the Mitra-Clip Percutaneous Therapy for Heart Failure Patients with Functional Mitral Regurgitation), published in 2018. The MITRA-FR study enrolled 304 patients with HF, severe secondary $M R$, and impaired left ventricular ejection fraction (LVEF) (15\%-40\%) despite guideline-recommended optimal medical therapy and cardiac resynchronization therapy (CRT). Patients were randomized (1:1) to receive either TMVR or conservative treatment. The number of periprocedural complications was low. At discharge, 91.9\% of patients had a reduction in MR to $\leq 2+$. Regardless, there were no significant differences in the primary endpoint rate (death or unplanned hospitalization for HF) at 12 months (54.6\% vs. $51.3 \%)$. The mortality rate $(24.3 \%$ vs. $22.4 \%)$ or unscheduled hospitalization rate $(48.7 \%$ vs. $47.4 \%)$ did not differ significantly between the groups. Both groups achieved comparable symptomatic improvement (NYHA 
[New York Heart Association] class reduction at one year). Complete clinical data was provided on as many as $99 \%$ of patients. The limitation of the study was an incomplete echocardiographic follow-up [5].

The COAPT study enrolled 614 patients with moderate to severe secondary MR and HF (NYHA II-IV) despite the use of maximal doses of guideline-recommended medical therapy and implantation of CRT (36.5\%). The cause of cardiomyopathy was ischemic in $60.7 \%$ of the patients and nonischemic in $39.3 \%$. Patients were randomly assigned to the MitraClip-device group ( $n=302$ ) or medical therapy alone - the control group $(n=312)$. The study revealed a significantly lower rate of hospitalization for heart failure (35.8\% vs. $67.9 \%$; HR 0.53; $95 \% \mathrm{Cl}, 0.40-0.70 ; P<0.001$; $\mathrm{NNT}=3.1)$ and lower all-cause mortality $(29.1 \%$ vs. $46.1 \%$; HR 0.62; 95\% Cl, 0.46-0.82; $P<0.001$ ) within 24 months of follow-up in the patients with device-based treatment compared with medical therapy alone. Moreover, their quality of life was significantly better, functional capacity was more preserved, and mitral regurgitation and left ventricular end-diastolic volume were reduced (secondary endpoints) [6].

The discrepancies between the results of both abovementioned trials may be due to heterogeneous inclusion criteria and direct effects of the procedure (Table 1), which requires a comment. The studied groups differed in sample size (304 vs. 614 patients) and the duration of clinical follow-up for the primary endpoint (12 vs. 24 months).

In COAPT, the primary endpoint was hospitalization for HF, while in MITRA-FR, it was a composite endpoint (all-cause mortality and unscheduled hospitalization for HF). Inclusion criteria and MR definition were also different (MITRA-FR: effective regurgitant orifice area [EROA] $\geq 20 \mathrm{~mm}^{2}$ and/or regurgitant volume $>30 \mathrm{ml}$; COAPT: $E R O A \geq 30 \mathrm{~mm}^{2}$ and regurgitant volume $>45 \mathrm{ml}$ ), as well as left ventricular ejection fraction (LVEF) (MITRA-FR: $15 \%-40 \%$; COAPT: $20 \%-50 \%)$, and end-systolic left ventricle diameter (COAPT: $<70 \mathrm{~mm}$, lack of this criterion in MITRA-FR). As a result, in the MITRA-FR study, the mean left ventricular volume was higher $\left(135 \mathrm{ml} / \mathrm{m}^{2}\right.$ vs. $\left.101 \mathrm{ml} / \mathrm{m}^{2}\right)$, and the mitral regurgitation was less severe (EROA $31 \pm 10$ vs. $41 \pm 15 \mathrm{~mm}^{2}$ ), with comparable LVEF values ( $33 \pm 7$ vs. $31 \pm 7 \%$ ). Therefore, in the COAPT trial, the percentage of patients with severe $M R\left(E R O A \geq 40 \mathrm{~mm}^{3}\right)$ was significantly higher (41 vs. $\left.16 \%\right)$. Although the percentage of successful procedures in the MITRA-FR study was high, it was significantly higher in the COAPT study. Inclusion in the COAPT study required optimization of pharmacological therapy, confirmed by an independent team of experts prior to enrolment. MITRA-FR imposed less strict inclusion criteria, which is more similar to "real-world" practice [7]. Detailed post-hoc analyzes indicated that the inclusion and exclusion criteria for both trials led to the COAPT study enrolling patients with clinically predominant MR as the major mechanism of heart failure. In contrast, the MITRA-FR study included more patients with severe left ventricular dysfunction and a relatively less severe MR as a major cause of HF. Results of the extended 3-year follow-up of patients in the COAPT study confirmed the effectiveness of TMVR with the MitraClip system in reducing mortality and rehospitalization rates and improving the quality of life [8].

From a practical point of view, patients enrolled by the multidisciplinary Heart Team for MitraClip TMVR should meet the criteria of the high probability of a favorable response to this type of treatment.

The ongoing Reshape-HF2 trial (A Clinical Evaluation of the Safety and Effectiveness of the MitraClip System in the Treatment of Clinically Significant Functional Mitral Regurgitation [Reshape-HF2], https://clinicaltrials. gov/ct2/show/NCT02444338) has similar eligibility criteria in terms of MR as the COAPT study and intermediate criteria between COAPT and MITRA-FR for the assessment of left ventricular dysfunction.

To date, we have not obtained any results of randomized clinical trials comparing the effectiveness of transcatheter and surgical repair of mitral valve insufficiency in patients with secondary (functional) MR. The evaluation of these two strategies is the subject of an ongoing MATTERHORN study (Multicenter, Randomized, Controlled Study to Assess Mitral vAlve reconsTrucTion for advancEd Insufficiency of Functional or iscHemic ORigiN).

Table 1. Similarities and differences between MITRA-FR and COAPT studies with respect to echocardiographic characteristics and procedural outcomes immediately and at one year post-procedure

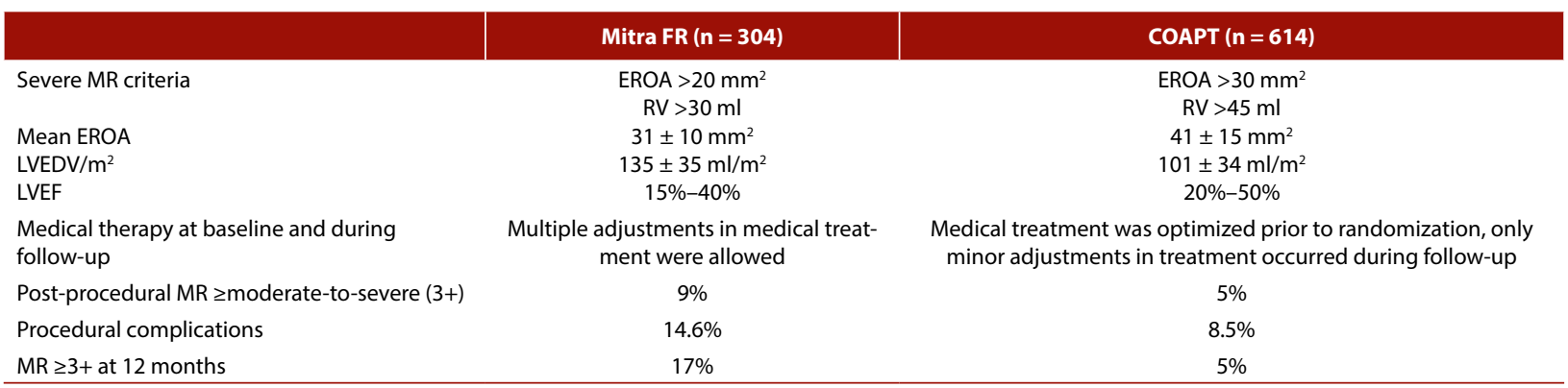

Abbreviations: COAPT, Cardiovascular Outcomes Assessment of the Mitra-Clip Percutaneous Therapy for Heart Failure Patients with Functional Mitral Regurgitation; EROA, effective regurgitant orifice area; LVEDV, ventricular end-diastolic volume; LVEF, left ventricular ejection fraction; MITRA-FR, Percutaneous Repair with the MitraClip Device for Severe Functional/Secondary Mitral Regurgitation; MR, mitral valve regurgitation; RV, regurgitant volume 


\section{Registries}

Important data on edge-to-edge TMVR comes from registers - pan-European ACCESS-EU and German TRAMI. In the ACCESS-EU registry, approximately $70 \%$ of patients had secondary MR. MitraClip procedures were highly effective (99.6\%) and safe (30-day mortality 3.4\%). Additionally, after 6 months, $80 \%$ of patients achieved MR reduction to $\leq 2+$ and symptomatic improvement to NYHA class I/II. The 6-month mortality rate was $11.2 \%$. Data from the TRAMI registry of 486 patients indicate low (2.5\%) in-hospital mortality and $12.5 \%$ mortality after three months $[9,10]$. Expanding the anatomical indications for the procedure beyond those applicable in the EVEREST trial does not reduce the effectiveness of this method.

The Getting Reduction of Mitral Insufficiency by Percutaneous Clip Implantation (GRASP) registry compared patients who met the EVEREST criteria with those whose mitral valve anatomy was less favorable. It has been demonstrated that in both groups, incidence rates of the composite endpoint were similar, which indicates that it is justified to extend the inclusion criteria to inoperable/highrisk patients for whom the procedure is technically feasible. Similarly, the MitraSwiss, TRAMI, and Taramasso et al. registries covering patients with primary and secondary MR showed that the long-term prognosis in the groups that did not strictly meet EVEREST criteria was not inferior to the patients who did [10-12].

Increasing experience with the MitraClip system, especially in centers with a trained team and sufficient experience ( $\geq 50$ procedures), shows that edge-to-edge repair may be a safe alternative to surgery in selected cases. However, the MR recurrence rate is significantly higher than in the case of surgical treatment.

A meta-analysis that included 1015 patients from 7 studies comparing both techniques has shown comparable 30-day and 6-month mortality rates despite higher EuroSCORE in the transcatheter group. Nevertheless, the severe MR recurrence rate was almost five times higher in the TMVR group [13]. It should be emphasized that recurrent $M R$, as well as residual regurgitant jet size, are strong predictors of long-term mortality in patients after mitral valve repair procedures in both primary and secondary mitral regurgitation [1].

Clinical experience with the PASCAL system (another edge-to-edge TMVR system) includes registry studies from European heart valve centers. They indicate the effectiveness of this system in reducing MR and functional improvement of HF, mainly in patients with secondary MR [14]. The PASCAL system has recently been registered in Poland.

\section{Recommendations of scientific societies}

Newest ESC/EACTS Guidelines on the management of valvular heart disease had been published in 2021, so they reflect the progress of knowledge on transcatheter TMVR in patients with secondary MR. 2017 valvular heart disease guidelines included only the EVEREST and EVEREST
II findings, which served as the basis for the approval of the MitraClip system in the US in patients with primary MR. 2021 Guidelines expand the indication to patients with heart failure and secondary MR in accordance with the results of the randomized COAPT and MITRA.Fr studies. The guidelines reinforce the heart team-based approach to patients' selection and qualification for edge-to-edge repair. Preferably the patients should fulfill the clinical and anatomic criteria compatible with the COAPT (high likelihood of improvement). The class of indications is Ila with level of evidence (LOE) B for patients not eligible for surgery and with high likelihood of improvement and IIb LOE C in selected high-risk symptomatic patients not eligible for surgery and not meeting the criteria of increased chance of response to edge-to-edge repair [15]. In general the guidelines pave the way to more widespread use of the edge-to-edge treatment in symptomatic HF patients with the aim to reduce the symptoms burden and heart failure rehospitalizations.

The American College of Cardiology/American Heart Association's guidelines for the management of patients with valvular heart disease published in 2021 include new, less restrictive recommendations for edge-to-edge TMVR, which extend the pre-existing indications, in the case of 1) severe primary MR in symptomatic patients (NYHA class III-IV) with high or prohibitive surgical risk, favorable valve anatomy and estimated survival time over one year (class of recommendation Ila); 2) severe secondary MR associated with left ventricular dysfunction (LVEF $<50 \%$ ), symptoms in functional classification II-IV despite optimal pharmacotherapy of $\mathrm{HF}$, favorable valve anatomy on echocardiography, LVEF $20 \%-50 \%$, left ventricular end systolic diameter $\leq 70 \mathrm{~mm}$ and pulmonary artery systolic pressure $\leq 70 \mathrm{~mm} \mathrm{Hg}$ (class of recommendation Ila) [16]. If coronary revascularization is not necessary, the recommendations for surgical treatment in this particular group of patients are class IIb. The classification algorithm for transcatheter mitral valve procedures based on the American Heart Association/American College of Cardiology's guidelines is presented in Figure 1.

\section{Determining patients' eligibility for TMVR}

The role of the multidisciplinary Heart Team in determining proper treatment strategies Decision-making for intervention should be made by a "Heart Team" with particular expertise in valvular heart disease, comprising cardiologists, cardiac surgeons, interventional cardiologists, anesthetists, and imaging specialists, experienced in mitral valve anatomy assessment and interventional imaging in structural heart disease. In some cases, the professional opinion of a radiologist performing multislice computed tomography of the heart is necessary.

Due to the high risk associated with comorbidities, patients should be treated in centers with highly specialized multidisciplinary teams, trained in both surgical and tran- 


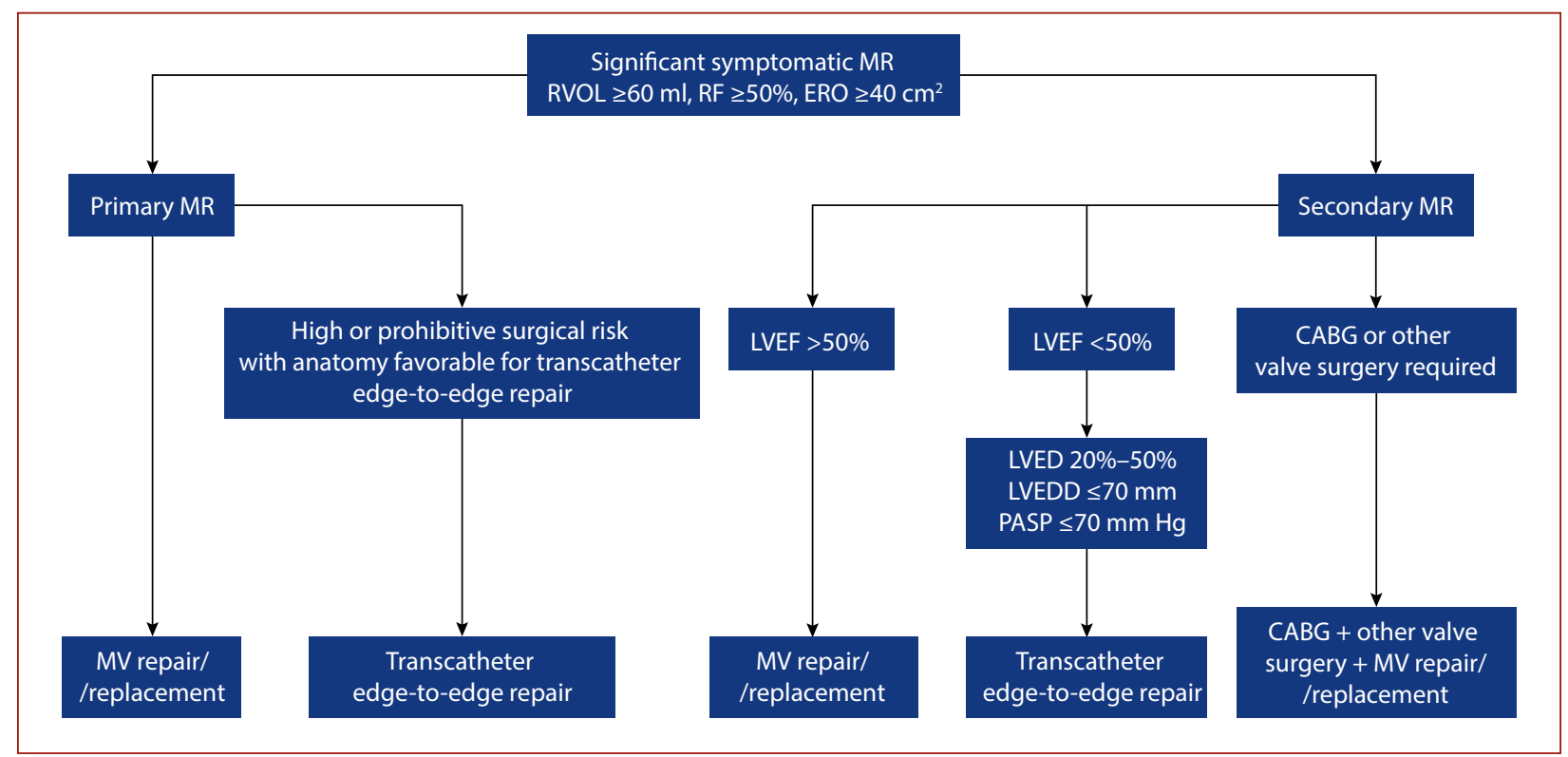

Figure 1. Algorithm for management of patients with significant symptomatic MR. Based on: [15] and [16].

Abbreviations: CABG, coronary artery bypass grafting; other — see Table 1

scatheter procedures to deliver high-quality care ("heart valve centers"). In addition to an interventional cardiologist or a cardiac surgeon experienced in a given interventional technique, a cardiologist experienced in procedural transesophageal echocardiographic imaging plays an important role in a minimally invasive valve repair or implantation procedure because, unlike other transcatheter procedures (e.g., TAVI), the use of fluoroscopy is of less importance and proper planning of the procedure and surgical navigation are possible only based on a transesophageal examination, optimally with three-dimensional imaging. Patients treated with TMVR should undergo a periodic clinical and echocardiographic evaluation to exclude late complications and determine long-term efficacy. The Heart Team's opinion is of particular importance in patients with primary MR, in whom conventional surgical treatment is the procedure of choice, and the referral for transcatheter treatment applies to the surgically ineligible patients. In the group of patients with secondary MR, it is crucial to provide optimal pharmacological therapy in accordance with the guidelines and, if applicable, implantation of CRT. Patients eligible for the transcatheter procedure are those with symptomatic HF despite pharmacotherapy optimization. Cooperation with HF specialists may be helpful to facilitate patient management and to decide on heart transplant or left ventricular assist devices (LVAD) implantation in uncertain cases.

\section{Clinical evaluation}

1. Symptomatic HF (NYHA II-IV) despite optimal medical pharmacotherapy in maximum tolerated doses, in line with the guidelines for the management of heart failure, including CRT implantation if indicated, as well as complete revascularization;
2. Shared decision of the multidisciplinary heart team on TMVR. In patients with primary MR, edge-to-edge TMVR can be considered for inoperable or high-risk surgical patients. However, in the case of secondary MR, the edge-to-edge repair is the treatment of choice in patients meeting clinical and anatomical criteria who are not candidates for surgical revascularization. Transcatheter edge-to-edge repair may also be beneficial in patients waiting for a heart transplant or LVAD implantation;

3. Estimated life expectancy should be at least 12 months in patients with primary MR. In patients with secondary $M R$, the futility of interventions in patients unlikely to benefit from the treatment must be taken into account;

4. It is recommended to avoid surgery in patients with a low chance of improved expected quality of life due to their general condition (emaciation, advanced frailty syndrome), and comorbidities. Important determinants include the presence of severe pulmonary hypertension, severe right ventricular dysfunction with significant tricuspid regurgitation, extremely high NT-proBNP levels ( $>5000 \mathrm{pg} / \mathrm{ml}$ ), and LVEF $<20 \%$. The coexistence of pulmonary hypertension, right ventricular dysfunction, and LVEF $<20 \%$ drastically worsens the prognosis;

5. Optimal pharmacotherapy.

Since the COAPT study confirmed the benefit of edgeto-edge repair treatment in patients for whom optimization of pharmacotherapy was an essential element of referral for the procedure, it is recommended that all implanting centers should assess the pharmacotherapy in the screening protocol following ESC recommendations.

It is recommended to consider the following drug groups: (1) angiotensin-converting-enzyme inhibitors/an- 
giotensin II receptor antagonist or sacubitril/valsartan; (2) a beta-blocker in an optimal dose - with dose adjustments based on the patient's heart rate and blood pressure; (3) mineralocorticoid receptor antagonists; (4) loop diuretic; (5) ivabradine. The abovementioned pharmacotherapy should be continued for a minimum of 3 months in an outpatient clinic before deciding on the referral. The ineffectiveness of the therapy or lack of the possibility of its further optimization should be documented.

\section{Echocardiographic criteria}

Patients' referrals for procedures performed without extracorporeal circulation are based on the information obtained by three-dimensional (3D) transthoracic and transesophageal echocardiography. Echocardiographic evaluation should provide information on 1) the severity and mechanism of MR; 2) the validity of the procedure based on the MR assessment in relation to the volume of the left ventricle, by dividing patients into groups with "proportionate" and "disproportionate" MR in patients with secondary regurgitation; 3 ) anatomical assessment of the mitral valve and subvalvular apparatus in terms of feasibility of the procedure; 4) evaluation of other parameters influencing the long-term outcomes of the procedure (left and right ventricular function, presence of other valvular diseases, including tricuspid valve regurgitation, presence of pulmonary hypertension); 5) contraindications.

1. Primary MR

a) Presence of severe MR based on qualitative, semi-quantitative, and quantitative assessments

b) No anatomical contraindications

\section{Secondary MR}

a) Presence of severe MR based on qualitative, semi-quantitative, and quantitative parameters assessment - at least moderate to severe MR as assessed with qualitative parameters (EROA $\geq 0.3 \mathrm{~cm}^{2}, E F \geq 40 \%$ ). Due to the asymmetric nature of the regurgitant orifice, quantitative assessment should be based, if possible, on a 3D reconstruction, and where 3D techniques are not available, an integrated approach is strongly recommended instead of referring to single measurements. Imaging should take into account vena contracta measured in two perpendicular planes, including along the coaptation line.

b) Disproportionate MR should be evaluated as EROA to the left ventricular end-diastolic volume (LVEDV) ratio (the higher the EROA/LVEDV $\left[\mathrm{mm}^{2} / \mathrm{ml} \times 100\right]$ ratio, the greater the profitability of the procedure compared to pharmacotherapy) or regurgitant volume to EDV ratio (RV/LVEDV). The left ventricular volume alone and LVEF (especially $<20 \%$ ) are also important prognostic factors for the lack of clinical improvement. Additionally, the presence of global vs. regional wall motion abnormalities, presence of symmetrical vs. asymmetrical regurgitant jet is assessed. This concept facilitates the decision-making process, but it is not the only parameter that should be taken into consideration. Simply put - it is reasonable to consider patients as eligible for the procedure, with LVEF $\geq 20 \%$ and left ventricular end systolic diameter $\leq 70 \mathrm{~mm}$; however, these parameters, especially regarding the end-diastolic dimension of the left ventricle, should be treated as indicative only and should be interpreted depending on the clinical condition, the severity of regurgitation, nature of left ventricular remodeling and the morphology of the valve itself.

c) Shared echocardiographic criteria for primary and secondary MR

- Primary morphological criteria for patient eligibility were based on a pre-specified EVEREST study protocol. Key inclusion criteria included a regurgitant jet origin associated with the abnormal coaptation of $\mathrm{A} 2$ to $\mathrm{P} 2$ segments, and the mitral valve area was equal to or greater than $4.0 \mathrm{~cm}^{2}$. Currently, it is more common to use the criteria which determine the feasibility of the intervention based on extensive experience and new surgical techniques, mainly from German and American centers. The evolution of the MitraClip system is also significant (introducing two lengths and widths of the clip arms and the possibility of independently gripping the leaflets in the G4 system). Nevertheless, mitral stenosis following Mitra-Clip insertion remains the primary concern, particularly in patients who require more than one clip due to broad regurgitant jet. Published in 2013, an expert opinion of the international group of experienced operators and echocardiography specialists divide patients into three groups in terms of mitral valve (MV) morphology:

- Ideal valve morphology for a MitraClip procedure: MR originating from the mid-portion of the valve (A2P2), no evidence of calcification in the grasping area of the A2 and/or P2 scallops, mitral valve area $>4.0 \mathrm{~cm}^{2}$ (3D-echo planimetry measurements are the first-choice method), posterior leaflet mobile length $\geq 10 \mathrm{~mm}$, coaptation depth $<11 \mathrm{~mm}$, a width of the flail segment $\geq 15 \mathrm{~mm}$, or flail gap $\geq 10 \mathrm{~mm}$.

- Unsuitable valve morphology for a MitraClip procedure: Severe calcification in the grasping area, perforated mitral leaflets or clefts, increased risk of postoperative mitral stenosis (mitral valve area $<3.5-4.0 \mathrm{~cm}^{2}-$ EVEREST exclusion criterion, which is not currently a key decision parameter), length of posterior leaflet $<7 \mathrm{~mm}$, Barlow's disease with multiple mitral regurgitation jets, pres- 
ence of a broad jet especially in patients with intermediate mitral valve area at baseline, which may indicate the need for multiple clips in order to successfully approximate leaflets and increase coaptation, rheumatic valve disease - with restriction in systole and diastole or endocarditic valve disease. The use of longer clips and the ability to independently grasp the leaflets make the limitations resulting from a large coaptation gap less significant.

- Complex: intermediate morphology between $a$ and $b$. The feasibility of the procedure depends on the experience of the center and the operators.

- The exclusion criteria for the MitraClip therapy are the evidence of intracardiac, inferior vena cava or femoral venous thrombus, cardiac tumor, or active endocarditis. Understanding the interatrial septum anatomy is of great importance. The presence of interatrial masses, such as an aneurysm, lipomatous hypertrophy of the interatrial septum, and patent foramen ovale, makes the procedure more challenging.

The feasibility of the procedure in other cases depends mainly on the experience of the operators and the quality of periprocedural imaging [17]. The procedure can be performed safely in patients who previously underwent transcatheter left atrial appendage occlusion.

\section{Recommendations}

Determining patients' eligibility for edge-to-edgeTMVR should be in alignment with the consensus recommendations developed in centers with expertise in both surgical and percutaneous treatment of mitral valve disease by an experienced multidisciplinary team. It is the first-choice treatment in patients with severe degenerative MR and groups with high or prohibitive surgical risk. In patients with secondary MR and HF, TMVR may be considered as long as a medical therapy has already been dose-optimized and CRT-D has been implanted, if indicated.

As the COAPT clinical trial consistently demonstrated the benefits of MitraClip in patients with secondary MR, it is advisable to select patients whose clinical and anatomical characteristics are similar to the COAPT eligibility criteria, and the likelihood of symptomatic improvement is high.

\section{Planning the procedure and post-operative care}

\section{Procedural aspects}

The procedure is performed under fluoroscopy and with continuous transesophageal echocardiography (TEE) guidance with 3D imaging. It is imperative to establish means of communication about the anatomy of the heart that is understood by the entire team (nomenclature of mitral valve [MV] segments, directions of movement [medial-lateral, anterior-posterior]).
TEE is crucial, from selecting the transeptal puncture site (fossa ovalis, approximately $4 \mathrm{~cm}$ above the mitral annulus and at a safe distance from the aorta and free atrial wall) to releasing the last clip. The site of transseptal puncture depends on the origin of mitral regurgitation jet (for medially directed jet, it is more beneficial to puncture the septum lower, in the bicaval projection, than for jet directed laterally). After a septal puncture, it is necessary to confirm the safe location of the guidewire in one of the pulmonary veins and during the navigation of the MitraClip device to confirm that it does not touch the atrium walls. If it is not feasible to insert a guidewire into the pulmonary vein (e.g., a significantly enlarged atrium), a pre-shaped stiff guidewire (e.g., Safari or similar) may be used and left behind in the atrium cavity until the guiding catheter is inserted. Optimal 2D imaging is usually feasible by tilting the TEE probe backward and placing it in the axis of the mitral valve and the left ventricle. The same axis should also be the trajectory of the clip insertion into the left ventricle, which is achieved by the proper introduction of the delivery system into the septum, its rotation, and setting the catheter deflection control knobs. Deviations from these rules usually cause difficulties in imaging the clip, skewed trajectory when passing the valve (diving), and subsequently uneven grasp of the leaflets, leading to an increased risk of valve deformation after the release of the device. The technique which facilitates and shortens this stage of the procedure is the intraoperative real-time 3D TEE imaging (RTTEE3D). The surgical view of the MV in RTTEE3D allows the maneuvers to position the clip arms perpendicularly to the line of coaptation at the intended implantation site. The crossing of the valve is usually done under the guidance of 2DTEE, preferably in 2 perpendicular planes (X-plane). After entering the ventricle, the position of the clip is once more assessed with RTTEE3D. The evaluation allows determining as well if the clip orientation is perpendicular to the line of MV coaptation.

The introduction of a new generation of MitraClip devices, including wider clips, allows obtaining a reduction of MR after a single clip application in a significant percentage of cases. However, in the presence of a broad jet, which requires multiple clips, it is reasonable to use the first one medially and the next ones laterally, which allows avoiding subsequent implantation in close proximity to the commissure.

To grasp the leaflets of the mitral valve, a 3-chamber mid-oesophageal view is sufficient, in which a cross-section through the arms of the device is visible simultaneously with the anterior and posterior leaflet (left ventricular outflow tract [LVOT] view). However, it is optimal to use two perpendicular views (three-chamber and two-chamber mid-oesophageal view - LVOT and intercommissural view), which allows for proper navigation, as well as determining when the clip is adequately positioned and whether arm orientation is perpendicular to the line of MV coaptation. 
The decision to release the clip is made on the basis of:

1. Improved mitral valve coaptation/ reduced size of mitral regurgitation jet;

2. The stability of the device judged by the length of the leaflets grasped by its closed arms and compared with their pre-treatment length;

3. Mean MV pressure gradient $<5 \mathrm{~mm} \mathrm{Hg}$.

Echocardiographic 3D-guided planimetry of the resulting double valve orifices is also helpful in the decision-making process.

During the procedure, significant manipulations should be avoided to minimize chordal and subchondral entanglement, which may damage the mitral subvalvular apparatus. The decision on multiple clip implantation depends on the MR reduction grade after the first MitraClip device deployment, the width of the baseline coaptation defect, the transvalvular gradient, and the presence of the clefts. Recently available clips with wider grasping areas allow reducing the number of clips required. Until the clip is released from the delivery system, it can be repositioned as needed. After the MitraClip system has been deployed and released, residual regurgitation and stenosis are assessed (with a mean gradient $\leq 5 \mathrm{~mm} \mathrm{Hg}$ and heart rate $\leq 80$ beats/min) as well as clip stability and presence of excessive pericardial fluid. 3D planimetry is also a useful tool in assessing the residual mitral valve area. The entire procedure should be preceded with a detailed analysis of each stage and in an agreement between the operator and interventional imaging cardiologist. It is of utmost importance to obtain an optimal MR reduction to provide better outcomes.

\section{Type of anesthesia}

MitraClip implantation procedures are performed under general anesthesia. It enables a short-term respiratory arrest and facilitates positioning of the system and grasping the leaflets of the valve in certain cases of high respiratory mobility. Recently published data suggest the safety of MitraClip procedures under deep analgosedation instead of general anesthesia, but this is not yet the recommended standard of practice [18]. Furthermore, general anesthesia improves patients' tolerance to the TEE probe placement in the supine position and ensures complete immobilization, which is crucial for the precision of the procedure.

\section{Hemostasis}

Due to the large-caliber delivery system (24F diameter; $8.1 \mathrm{~mm}$ ), appropriate hemostasis management is required. The following techniques are available: (1) prolonged manual compression; (2) "8" or "Z" suture, as well as; (3) off-label use of the pre-closure technique (1-2 Proglide sutures); (4) combining techniques 1-3.

No prospective studies are aiming to compare the efficacy of these different methods [19].

\section{Anticoagulant and antiplatelet therapy}

Activated Clotting Time (ACT) should be monitored regularly during the procedure. Target, therapeutic ACT level above $250-300$ seconds should be maintained. The timing of heparin administration varies. Most operators administer the full dose prior to transseptal puncture (TSP), others wait until puncture has occurred or administer half of the dose prior to puncture and the other half after TSP. The optimal time to initiate anticoagulation has not been studied so far. There is no reliable scientific evidence that would enable the formulation of clear recommendations regarding anticoagulant and antiplatelet treatment after the procedure. In patients without indications for anticoagulation, dual antiplatelet therapy with acetylsalicylic acid for 6 months is used, together with clopidogrel for the first month. Oral anticoagulation (vitamin $\mathrm{K}$ antagonist or non-vitamin $\mathrm{K}$ antagonists oral anticoagulant) is required in patients with atrial fibrillation. An individual bleeding risk assessment is required. Each center should develop an antiplatelet treatment protocol and include the recommendations on the patient's hospital discharge form.

\section{Hemodynamic assessment}

Along with echocardiographic assessment, additional information is provided by monitoring mean left atrial pressure (mLAP). No decrease or increase in mLAP after surgery is associated with a higher risk of readmission in a long-term follow-up for $\mathrm{HF}$, regardless of residual MR on TEE [20]. It is reasonable to measure mLAP during edgeto-edge repair procedures to evaluate the effectiveness of the intervention and assess the prognosis. A postoperative evaluation of pulmonary venous flow reversal as an indirect parameter of the effectiveness of the procedure is also important.

latrogenic atrial septal defect after transcatheter mitral valve repair

MitraClip placement requires interatrial transseptal puncture (IAS), which, due to the relatively large delivery system and guiding catheter ( $\geq 22 \mathrm{~F}$ ), creates an atrial septal defect (ASD). It may close spontaneously or remain patent after the procedure (incidence $50 \%-85 \%$ after 30 days and $<30 \%$ after 12 months, defects $<7-8 \mathrm{~mm}$ are more likely to close spontaneously). Factors favoring the formation of iatrogenic ASD (iASD) include the diameter of the delivery system, long surgery duration, multiple manipulations with the delivery system, left ventricular hypertrophy, delivery system maneuvers, and an increased postoperative left atrial pressure.

In terms of hemodynamic consequences, postoperative ASD may cause:

1. Acute complications immediately after the procedure: a severe left-to-right shunt with hypoxemia and acute heart failure (approximately $1.5 \%$ of patients);

2. Chronic complications associated with a left-to-right or bi-directional shunt (right ventricular overload, pulmonary hypertension);

3. Pressure relief of the left atrium with no negative impact;

4. No hemodynamically significant consequences. 
The right-to-left shunt is associated with a subsequent worse 12-month prognosis than the left-to-right shunt.

In case of acute respiratory failure, it is recommended to close the iASD immediately after the TMVR procedure with a dedicated occluder, using TEE guidance to assess the size of iASD. The long-term approach to postprocedural ASD with a persistent hemodynamic effect ( $L-R$ shunt) is controversial. The data from the registries are contradictory and show both the beneficial effect of left atrial decompression on $\mathrm{HF}$ symptoms, as well as the progression of HF and worsening of prognosis [21]. The randomized MITHRAS trial, in which the primary endpoint was changed in the 6-minute walk test distance, did not show transcatheter iASD closure superiority over conservative therapy in terms of functional outcomes [22].

\section{Summary}

Routine closure of the iASD is not recommended in patients after edge-to-edge repair procedures unless there is hemodynamic instability and periprocedural hypoxemia.

The decision to close the defect in an elective procedure depends on the hemodynamic significance of the shunt (right ventricular overload, pulmonary hypertension) and the risk of paradoxical embolism (venous thromboembolism, a history of pulmonary embolism). Operators should be acquainted with the ASD closure procedure; different sizes of ASD occluders ought to be available in the laboratory.

Procedural Complications and Complication Management

Periprocedural complications include:

- $\quad$ perioperative death $(<2 \%)$;

- bleeding at the access site of various severity, including major bleeding requiring transfusion (to 17.2\%) [23];

- early partial leaflet detachment (1\%-4.8\%);

- clip embolization $(<0.05 \%)$;

- leaflet perforation, mitral chordae rupture $(0.8 \%)$;

- cardiac perforation and pericardial tamponade (0.7\%);

- thrombus formation within the left atrium (approx.9\%);

- stroke and transient ischemic attack (0.9\%-1.3\%);

- renal failure (4.2\%);

- oesophageal damage $(0.6 \%-2.8 \%)$;

- gas embolism.

The formation of an atrial thrombus and/or on the device is an indication for prolonged heparin therapy in therapeutic doses and echocardiographic control prior to hospital discharge. Once the thrombus resolves, the use of an oral anticoagulant and regular echocardiographic monitoring should be considered.

In a small percentage of cases, patients with transcatheter treatment failure require urgent cardiac surgery. The incidence is rare $(<0.5 \%)$, and the number of cases decreases with the increasing experience of operators and centers [24]. In case of unsatisfactory MR reduction, dislocation of the clip, or relevant postprocedural mitral stenosis, surgical treatment should be considered. Complete clip detachment or clip embolization usually requires conventional surgery. Partial leaflet detachment of the clip may occur in the periprocedural period or after the procedure. Half of the patients with late MitraClip single leaflet detachment are treated conservatively. In case of unsuccessful initial repair and development of severe recurrent MR, a repeated MitraClip procedure is a feasible treatment option for high/prohibitive risk patients. In some patients, it is necessary to consider surgical treatment if the placement of the clip causes ischemia.

\section{Postprocedural clinical assessment}

Transthoracic echocardiography evaluation prior to hospital discharge is recommended. Echocardiographic reassessment after approximately 30 days, 6 months, and 1 year is justified.

It is useful to evaluate the volume of the left ventricle, the size of the left atrium, and the pulmonary venous flow (indirect parameters indicative of a permanent reduction of the regurgitant jet), as well as the size of the iASD, right ventricular function, and pulmonary hypertension. During the postoperative evaluation, it is also necessary to perform routine laboratory tests, optimize pharmacotherapy, and evaluate the compliance with guideline-recommended anticoagulant and/or antiplatelet therapy.

\section{Alternative uses of MitraClip system (early stages of clinical testing)}

Limited data from the registers suggest safety and efficacy of MitraClip procedures in MR correction in selected patients from the following groups: patients considered for orthotopic heart transplantation $(\mathrm{OHT})$ or implantation of a left ventricular assist device (LVAD) as a bridge procedure providing hemodynamic support; in patients with residual regurgitation jet after the surgical mitral valve repair; in patients with severe symptomatic MR related to obstructive hypertrophic cardiomyopathy to eliminate systolic anterior motion of the mitral valve; in therapy-resistant cardiogenic shock related to decompensated $\mathrm{HF}$ and concomitant severe chronic MR, and in patients with acute MR. There is no clinical data to support the long-term effectiveness of this approach. Decisions in such cases should be made by a multidisciplinary team [25]. Edge-to-edge procedures are increasingly performed in patients with concomitant or isolated tricuspid regurgitation in order to reduce the tricuspid regurgitant jet velocity $[2,3]$.

\section{Institutional and operator requirements}

Operator: It is recommended that the learning curve of operators and echocardiographers include the first 50 patients with optimal MV morphology, and then patients in the conditionally acceptable MV anatomy group may be treated.

Pursuant to the regulation of the Ministry of Health of November 12,2015 , specifying the conditions for highly specialized services, transcatheter non-surgical repair of the mitral valve in high-risk patients may be performed 
in centers that meet, among other things, the following requirements: (1) hybrid cardiac catheterization laboratory; (2) interventional radiology or catheterization laboratory; (3) intensive postoperative care in conditions equivalent to intensive care. The team performing the procedure should have extensive documented experience in mitral valve repair (cardiac surgeon) or transcatheter treatment of structural heart disease (cardiologist), as well as an available specialist in echocardiographic imaging, an anesthesiologist, surgical nurses, and a perfusionist. Supervision over the patient after the procedure is performed, apart from the intensive care or intensive cardiac care unit staff, also by a team consisting of a cardiac surgeon, a cardiologist, and a nurse specializing in the field of anesthetic and intensive care nursing.

In addition, the regulation specifies requirements for the team's experience ( 10 transcatheter non-surgical mitral valve repair/replacement procedures in high-risk patients performed) as well as the eligibility criteria:

a) Patients with severe symptomatic MR (EROA $>0.3$ for functional and $>0.4$ for primary $M R$ );

b) Disqualified by a multidisciplinary team from classical (surgical) or minimally invasive surgical treatment due to a documented high cardiac surgery risk;

c) The patient selection process is performed by the heart team.

Procedures should be reported as a part of the National Registry of Cardiac Surgery Procedures - however, it seems advisable to create a dedicated register covering all transcatheter edge-to-edge procedures, enabling a reliable follow-up assessment of short and long-term results of the procedure.

\section{Percutaneous mitral valve procedures during COVID-19 pandemic}

The recently published expert opinion of the Working Group on Valvular Heart Diseases, the Working Group on Cardiac Surgery, and the Association of Cardiovascular Interventions of the Polish Cardiac Society emphasizes the need to reduce the risk of patients and staff's infection with SARS-CoV-2 virus. Simultaneously, it is crucial that patients with symptomatic HF, despite guideline-directed medical therapy, can receive interventional treatment of the MR, which may favorably affect their quality of life and prognosis [26].

\section{DEVICES FOR PERCUTANEOUS CORRECTION OF MITRAL REGURGITATION, WITH THE "CE" MARK, THE USE OF WHICH HAS NOT BEEN INCLUDED IN THE CURRENT VHD GUIDELINES}

Carillon mitral contour system (Cardiac Dimension Inc., Kirkland, WA, USA) is the only device for percutaneous indirect mitral valve annuloplasty which has obtained the "CE" mark. The device is intended for the treatment of pa- tients with functional MR. The Carillon device incorporates two self-expanding anchors and a pre-shaped connecting bridge segment. The implantation procedure is performed using a venous approach under general anesthesia under the guidance of TEE. When delivered percutaneously to the coronary sinus, the Carillon device causes a decrease in the mitral annulus size and subsequently significantly reduces the mitral regurgitant volume. If the obtained effect is suboptimal or the compression of or the obstruction to the flow in the circumflex coronary artery or its branches is observed, the device can be folded and removed. AMADEUS (CARILLON Mitral Annuloplasty Device European Union Study), TITAN (Tighten the Annulus Now), and TITAN II studies have shown that mitral annuloplasty using the Carillon system significantly improves the quality of life by reducing MR, as well as physical performance assessed with the 6-minute walk test in patients with FMR [27]. In 2019 , the results of a multicenter, blinded, randomized, sham-controlled REDUCE-FMR (Carillon Mitral Contour System for Reducing Functional Mitral Regurgitation) trial were published. One hundred twenty patients receiving optimal heart failure medical therapy were randomized to either the Carillon system implantation group for mitral annular reduction or the sham-controlled arm. At one year, a statistically significant reduction in mitral regurgitant volume in the treatment group compared to the control group (decrease of $7.1 \mathrm{ml} /$ beat vs. an increase of $3.3 \mathrm{ml} /$ beat, respectively; $P=0.049$ ), left ventricular end-diastolic volume decrease (of $10.4 \mathrm{ml} v$ s. an increase of $6.5 \mathrm{ml} ; P=0.03$ ), and left ventricular end-systolic volume decrease (of $6.2 \mathrm{ml}$ vs. an increase of $6.1 \mathrm{ml} ; P=0.04$ ) were observed. When implanting the Carillon device, it should be remembered that perforation of the thin-walled coronary sinus is a possible procedural complication and that in some patients, the circumflex coronary artery may be compressed with subsequent flow obstruction. For the above reasons, $14 \%$ of patients were not implanted with Carillon in the REDUCE-FMR study [28]. Indirect mitral annuloplasty using Carillon is an effective treatment method that reduces MR and results in favorable left ventricular remodeling. However, the device is now used relatively infrequently in daily clinical practice.

Cardioband mitral system (Edwards Lifesciences, Irvine, CA, USA) is intended for percutaneous, direct mitral valve annuloplasty in patients with functional MR. The procedure is performed under general anesthesia guided by TEE. The device is delivered through a femoral venous puncture. The most crucial part of the Cardioband is a polyester-covered wire, which is successively attached to the back of the patient's native mitral ring with a number of anchors, and after the insertion, it resembles an incomplete surgical ring. The mitral annulus is then reduced in size using a unique regulating tool under the TEE guidance to minimize MR. In 2019, a multicenter study was published, which demonstrated the impact of Cardioband directly after implantation and at 12 months in 60 patients with 
echocardiographically assessed moderate (27\%) or severe (73\%) secondary MR. Technical success was achieved in $97 \%$, device success in $72 \%$, and procedural success in $68 \%$ of patients. In addition, there was a significant reduction of the septolateral diameter $(3.7 \pm 0.4$ vs. $2.6 \pm 0.4 \mathrm{~cm} ; P<0.01)$. The mitral regurgitation grade (in alive patients free of reintervention) and at 12 months was mild in $69 \%$, moderate in $26 \%$, and severe in $5 \%$ of patients. Anchor disengagement was observed in 10 patients, resulting in device inefficacy in 5 patients. In none of these patients did the device migrate or detach from the mitral ring. In the follow-up, six patients required percutaneous MR correction. During Cardioband implantation, care should be taken not to injure a coronary artery with the anchor while securing the ring [29].

The Cardioband customized annular reduction procedure reduces MR; therefore, it can be assumed that role of Cardioband in the treatment of functional MR will significantly increase in the future.

TRANSCATHETER CHORDAL REPAIR IN MR Neochord DS 1000 (Neochord Inc., St. Louis Park, MN, USA) and Harpoon (MVRS, Edwards Lifesciences, Irvine, CA, USA) systems, which both received CE mark approval, allow transesophageal echocardiography-guided (2D and 3D) transapical, beating-heart MV repair with insertion of prosthetic chords. The Neochord system allows the implantation of artificial chords to the edges of MV leaflets by capturing the leaflets and their puncture with a needle. The Harpoon system obtains the leaflet capture via a double helix-shaped knot on the atrial aspect of the leaflet after its puncture by the integrated needle. In both systems, the length of the chords is adjusted under echocardiography guidance, and the apical end of the chords is secured on the external surface of the heart. In both methods, implantation of at least 3 chords is recommended. Neo-chord implantation is dedicated to patients fulfilling anatomical criteria, e.g. the sum of the leaflet lengths should be at least $20 \%$ larger than the anterior-posterior dimension of the mitral annulus to achieve the coaptation of $5 \mathrm{~mm}$. In the Neochord group, the best results were obtained in patients with elongated or ruptured chordae tendineae to the $\mathrm{P} 2$ segment of the posterior leaflet [30]. So far the Harpoon system has been used only in patients with P2 pathology.

Numerous observational studies have demonstrated the safety and effectiveness of both methods; therefore, they are already widely used clinically [31, 32]. The Harpoon device is equipped with a hemostatic introducer that minimizes intraoperative bleeding. Due to the lack of a dedicated hemostatic sheath, it is recommended to use the Cell Saver system with the Neochord technique. The validity of these techniques has been documented extensively in a 5-year follow-up. Initial reports on large groups showed that $10 \%-35 \%$ of patients have greater than moderate MR postoperatively, which requires careful selection of patients [33].

\section{TRANSSEPTAL TRANSCATHETER IMPLANTATION OF DEDICATED MITRAL VALVE BIOPROSTHESIS (UNDER CLINICAL TRIALS)}

Edge-to-edge MR correction procedures, although effective and safe in the majority of patients, are subject to numerous limitations. About $10 \%$ of patients have a significant residual in the postoperative evaluation. What is more, the use of the MitraClip/Pascal systems is technically not feasible in a number of candidates, and recurrent MR after the intervention remains a challenge. The learning curve for the MitraClip procedure flattens after 200 procedures. Therefore, it is justified to look for an alternative solution, such as percutaneous mitral valve implantation via the transseptal approach. Several systems are currently evaluated in preclinical and clinical trials. Use of some of the first-generation systems was discontinued, or the investigation has been temporarily suspended due to observed complications (e.g., valve thrombosis). TMVI is a far more complex procedure than TAVI due to the anatomy of the mitral valve, the risk of the obstruction of the LVOT, a larger size of the delivery systems, and sometimes difficulties in maneuvering the device in the left atrium. Furthermore, patients treated with TMVI often have multiple comorbidities, which result in a very high 30-day mortality ranging from $18 \%$ to up to $60 \%$ [4]. It should be noted that the papers published so far are based on a relatively small group of patients. At present, the prospect of routine trans-septal TMVI procedures is distant. The system described below was used in Poland in only a few eligible candidates.

High Life Valve (HighLife Medical Inc., Irvine, CA, USA) is a self-expanding bioprosthesis that consists of a nitinol alloy-based frame covered with a polyester graft and trileaflet bovine pericardium with an annular diameter of $28 \mathrm{~mm}$.

The first stage of the procedure is placing a ring-shaped implant through the aortic valve around the subvalvular apparatus. The ring ensures proper fixation for the prosthesis. Subsequently, a self-expanding prosthesis is delivered transseptally and implanted into the ring. So far, five successful procedures have been performed in Poland with the use of High-Life bioprosthesis. In total, approximately 30 treatments have been performed using this valve in the world. In preparation for the procedure, computed tomography plays a key role, which allows assessing the risk of LVOT obstruction, one of the basic eligibility criteria.

\section{IMPLANTATION OF DEDICATED TRANSCATHETER MITRAL VALVE BIOPROSTHESIS VIA TRANSAPICAL ACCESS}

Transapical TMVI has emerged to be a promising alternative to edge-to-edge repair. Transcatheter mitral valve replacement systems designed for transapical delivery, which are still evaluated in clinical trials but for which already quite extensive clinical experience is available, are Tendyne Bioprostehsis (Abbott Inc, USA), Intrepid Valve (Twelve Inc, Medtronic Inc., Fridley, MN, USA), CardiAQ (Edwards 
Lifesciences, Irvine, CA, USA), Tiara (Neovasc, Richmond, Canada), and Gate Valve (Navigate Inc., James Bay, CA USA). Some of the abovementioned have already received the CE mark (Tendyne), and others are still in the clinical trial phase. In a simplified manner, the procedural technique consists of gaining access to the left ventricle through a large dedicated delivery sheath and precise off-pump implantation (during rapid ventricular pacing) of a mitral valve bioprosthesis into the native mitral annulus or the leaflets of the patient's calcified native valve. Procedural guidance is mainly performed under fluoroscopy and TEE assistance.

TENDYNE valve is currently the only CE-approved and commercially available transcatheter mitral valve implant. However, unlike the previous valves, it is implanted via a transapical approach. The valve consists of 2 nitinol self-expanding joined stents with three porcine pericardial leaflets sewn into the frame. The system is anchored with the use of a tether and epicardial pad to the apex of the heart. In a registry of 100 patients with severe heart failure and reduced left ventricular ejection fraction, the technical success of the procedure was $96 \%$, the 30 -day mortality was $6 \%$, and the 1 -year survival rate was $72.4 \%$. Directly after the procedure, $98.9 \%$ of patients had none-trace mitral regurgitation, and $98.4 \%$ at one year [35].

\section{CONCLUSIONS}

In patients with severe symptomatic primary MR, valve surgery is the treatment of choice. In patients at high or prohibitive surgical risk, a catheter-based percutaneous edge-to-edge repair technique to correct mitral regurgitation has emerged as a feasible alternative therapeutic option. Patients with secondary MR and HF, despite optimal pharmacological therapy and CRT, benefit from edge-toedge procedures when the dominant mechanism of $\mathrm{HF}$ is mitral regurgitation rather than end-stage left ventricular dysfunction.

In both types of MR, referral for transcatheter treatment is a consensus of a multidisciplinary team consisting of a cardiac surgeon, an interventional cardiologist, a noninvasive cardiologist, an interventional imaging specialist, and an anesthesiologist. It is crucial to assess the predicted survival time and the probability of achieving an effective reduction of the regurgitation jet. It should be emphasized that LVAD implantation or OHT are highly effective treatment options for patients whose dominant symptom mechanism is heart failure and left ventricular damage. The results of the transcatheter treatment of MR should be quality controlled, preferably through a national registry. The population of patients requiring the minimally invasive transcatheter treatment because of the comorbidities and surgical risks is substantial. Access to such technologies in Poland is scarce and should be facilitated. The number of procedures is too low ( $<190$ procedures in 2020) to meet the clinical needs. The Valve-for-Life initiative of the European Association of Percutaneous Cardiovascular In- terventions supports the evidence-based implementation of transcatheter valve technologies in Poland (www.aisn.pl).

\section{Article information}

Conflict of interest: WW received lecture fees and grants from Abbott. AG received consultant fees and proctoring fees from Edwards Lifesciences and proctorig fees from Abbott. KB received consultant and proctoring fees from Edwards Lifesciences. PS received proctoring fees from Abbott. ZK received proctoring fees (LAAC) from Abbott. JT received proctoring fees from Abbott. PŚ received speaker's and proctor's fees Abbott. KW received proctoring fees from Neochord. GS received proctoring fees from Abbott. SB received leture fees from Abbott. AW received lecture fees from Abbott and Edwards Lifesciences. Other authors declared no conflicts.

Open access: This article is available in open access under Creative Common Attribution-Non-Commercial-No Derivatives 4.0 International (CC BY-NC-ND 4.0) license, allowing to download articles and share them with others as long as they credit the authors and the publisher, but without permission to change them in any way or use them commercially. For commercial use, please contact the journal office at kardiologiapolska@ptkardio.pl.

How to cite: Wojakowski W, Chmielak Z, Widenka K, et al., Transcatheter mitral valve repair and replacement. Expert consensus statement of the Polish Cardiac Society and the Polish Society of Cardiothoracic Surgeons. Kardiol Pol. 2021; 79(10): 1165-1177, doi: 10.33963/KP.a2021.0116.

\section{REFERENCES}

1. Petrus AHJ, Dekkers OM, Tops LF, et al. Impact of recurrent mitral regurgitation after mitral valve repair for functional mitral regurgitation: longterm analysis of competing outcomes. Eur Heart J. 2019; 40(27): 22062214, doi: 10.1093/eurheartj/ehz306, indexed in Pubmed: 31114862.

2. Orban M, Rommel KP, Ho EC, et al. Transcatheter edge-to-edge tricuspid repair for severe tricuspid regurgitation reduces hospitalizations for heart failure. JACC Heart Fail. 2020; 8(4): 265-276, doi: 10.1016/j. jchf.2019.12.006, indexed in Pubmed: 32241534.

3. Mehr M, Karam N, Taramasso M, et al. TriValve and TRAMI Investigators. Combined tricuspid and mitral versus isolated mitral valve repair for severe MR and TR: an analysis from the TriValve and TRAMI registries. JACC Cardiovasc Interv. 2020; 13(5): 543-550, doi: 10.1016/j. jcin.2019.10.023, indexed in Pubmed: 31954679.

4. Del Val D, Ferreira-Neto AN, Wintzer-Wehekind J, et al. Early experience with transcatheter mitral valve replacement: a systematic review. J Am Heart Assoc. 2019; 8(17): e013332, doi: 10.1161/JAHA.119.013332, indexed in Pubmed: 31441371.

5. Baumgartner H, Falk V, Bax JJ, et al. ESC Scientific Document Group. 2017 ESC/EACTS Guidelines for the management of valvular heart disease. Eur Heart J. 2017; 38(36): 2739-2791, doi: 10.1093/eurheartj/ehx391, indexed in Pubmed: 28886619.

6. Feldman T, Wasserman HS, Herrmann HC, et al. Percutaneous mitral valve repair using the edge-to-edge technique: six-month results of the EVEREST Phase I Clinical Trial. J Am Coll Cardiol. 2005; 46(11): 2134-2140, doi: 10.1016/j.jacc.2005.07.065, indexed in Pubmed: 16325053.

7. Feldman T, Foster E, Glower DD, et al. EVEREST II Investigators. Percutaneous repair or surgery for mitral regurgitation. N Engl J Med. 2011;364(15): 1395-1406, doi: 10.1056/NEJMoa1009355, indexed in Pubmed: 21463154.

8. Mack MJ, Lindenfeld J, Abraham WT et al. 3-Year Outcomes of Transcatheter Mitral Valve Repair in Patients With Heart Failure. J Am Coll Cardiol. 2021;77(8): 1029-1040, doi: 10.1016/j.jacc.2020.12.047, indexed in Pubmed: 33632476.

9. Feldman T, Kar S, Elmariah S, et al. EVEREST II Investigators. Randomized comparison of percutaneous repair and surgery for mitral regurgitation: 5-year results of EVEREST II. J Am Coll Cardiol. 2015; 66(25): 2844-2854, doi: 10.1016/j.jacc.2015.10.018, indexed in Pubmed: 26718672.

10. Obadia JF, Messika-Zeitoun D, Leurent G, et al. Percutaneous repair or medical treatment for secondary mitral regurgitation. $N$ Engl J Med. 2018; 379(24): 2297-2306, doi: 10.1056/nejmoa1805374, indexed in Pubmed: 30145927. 
11. Stone GW, Lindenfeld J, Abraham WT, et al. Transcatheter mitral-valve repair in patients with heart failure. N Engl J Med. 2018; 379(24): 2307-2318, doi: 10.1056/NEJMoa1806640, indexed in Pubmed: 30280640.

12. Pibarot $P$, Delgado V, Bax JJ. MITRA-FR vs. COAPT: lessons from two trials with diametrically opposed results. Eur Heart J Cardiovasc Imaging. 2019; 20(6):620-624, doi: 10.1093/ehjci/jez073, indexed in Pubmed: 31115470.

13. Maisano F, Franzen $\mathrm{O}$, Baldus $\mathrm{S}$, et al. Percutaneous mitral valve interventions in the real world: early and 1-year results from the ACCESS-EU, a prospective, multicenter, nonrandomized post-approval study of the MitraClip therapy in Europe. J Am Coll Cardiol. 2013; 62(12): 1052-1061, doi: 10.1016/j.jacc.2013.02.094, indexed in Pubmed: 23747789.

14. Kalbacher D, Schäfer U, V Bardeleben RS, et al. Long-term outcome, survival and predictors of mortality after MitraClip therapy: Results from the German Transcatheter Mitral Valve Interventions (TRAMI) registry. Int J Cardiol. 2019; 277: 35-41, doi: 10.1016/j.ijcard.2018.08.023, indexed in Pubmed: 30153994.

15. Gammie JS, Wilson P, Bartus K, et al. Transapical beating-heart mitral valve repair with an expanded polytetrafluoroethylene cordal implantation device: initial clinical experience. Circulation. 2016; 134(3): 189-197, doi: 10.1161/CIRCULATIONAHA.116.022010, indexed in Pubmed: 27436878.

16. Attizzani GF, Ohno Y, Capodanno D, et al. Extended use of percutaneous edge-to-edge mitral valve repair beyond EVEREST (Endovascular Valve Edge-to-Edge Repair) criteria: 30-day and 12-month clinical and echocardiographic outcomes from the GRASP (Getting Reduction of Mitral Insufficiency by Percutaneous Clip Implantation) registry. JACC Cardiovasc Interv. 2015; 8(1 Pt A): 74-82, doi: 10.1016/j.jcin.2014.07.024, indexed in Pubmed: 25499300.

17. Grasso C, Capodanno D, Rubbio AP, et al. One- and twelve-month safety and efficacy outcomes of patients undergoing edge-to-edge percutaneous mitral valve repair (from the GRASP Registry). Am J Cardiol. 2013; 111(10): 1482-1487, doi: 10.1016/j.amjcard.2013.01.300, indexed in Pubmed: 23433761.

18. Horn P, Hellhammer K, Minier M et al. Deep sedation vs. general anesthesia in 232 patients undergoing percutaneous mitral valve repair using the MitraClip ${ }^{\varpi}$ system. Catheter Cardiovasc Interv. 2017; 90(7): 1212-1219, doi: 10.1002/ccd.26884, indexed in Pubmed: 28112459.

19. Takagi $H$, Ando T, Umemoto T, et al. ALICE (All-Literature Investigation of Cardiovascular Evidence) Group. A review of comparative studies of MitraClip versus surgical repair for mitral regurgitation. Int J Cardiol. 2017;228: 289-294, doi: 10.1016/j.jijcard.2016.11.153, indexed in Pubmed: 27865200.

20. Besler C, Noack T, von Roeder M, et al. Transcatheter edge-to-edge mitral valve repair with the PASCAL system: early results from a real-world series. Eurolntervention. 2020; 16(10):824-832, doi: 10.4244/EIJ-D-20-00216, indexed in Pubmed: 32515739.

21. Yeo KK, Yap J, Tan JW, et al. Venous access closure using the double-proglide preclose technique after MitraClip implantation: long-term clinical and duplex ultrasound outcomes. J Invasive Cardiol. 2016; 28(2): 40-43, indexed in Pubmed: 26567455.

22. MitraClip provides valve repair without surgery. Harv Heart Lett. 2014; 24(6): 8, indexed in Pubmed: 24804351.

23. Schueler $\mathrm{R}$, Öztürk $C$, Wedekind JA, et al. Persistence of iatrogenic atrial septal defect after interventional mitral valve repair with the MitraClip system: a note of caution. JACC Cardiovasc Interv. 2015; 8(3): 450-459, doi: 10.1016/j.jcin.2014.10.024, indexed in Pubmed: 25703879.

24. Morikawa T, Miyasaka M, Flint N, et al. Right-to-Left shunt through iatrogenic atrial septal defect after MitraClip procedure. JACC Cardiovasc Interv. 2020; 13(13): 1544-1553, doi: 10.1016/j.jcin.2020.03.056, indexed in Pubmed: 32646695.

25. Kadado AJ, Islam A. latrogenic atrial septal defect following the MitraClip procedure: A state-of-the-art review. Catheter Cardiovasc Interv. 2021; 97(7): E1043-E1052, doi: 10.1002/ccd.29149, indexed in Pubmed: 32710470.

26. Lurz $P$, Unterhuber $M$, Rommel $K P$, et al. Closure of iatrogenic atrial septal defect after transcatheter mitral valve repair: the randomized MITHRAS trial. Circulation. 2021; 143(3): 292-294, doi: 10.1161/CIRCULATIONAHA.120.051989, indexed in Pubmed: 33054368.

27. von Bardeleben RS, Hobohm L, Kreidel F, et al. Incidence and in-hospital safety outcomes of patients undergoing percutaneous mitral valve edgeto-edge repair using MitraClip: five-year German national patient sample including 13,575 implants. Eurolntervention. 2019; 14(17): 1725-1732, doi: 10.4244/EIJ-D-18-00961, indexed in Pubmed: 30666965.

28. Isogai T, Saad AM, Shekhar S, et al. Incidence and short-term outcomes of surgical bailout after transcatheter mitral valve repair with the MitraClip system. Catheter Cardiovasc Interv. 2021; 97(2): 335-341, doi: 10.1002/ccd.29153, indexed in Pubmed: 32770712.

29. Thomas F, RaderF, Siegel RJ. The use of MitraClip for symptomatic patients with hypertrophic obstructive cardiomyopathy. Cardiology. 2017; 137(1): 58-61, doi: 10.1159/000454800, indexed in Pubmed: 28095388.

30. Płońska-Gościniak E, Suwalski P, Bartuś S, et al. Management of valvular and structural heart diseases during the coronavirus disease 2019 pandemic: an expert opinion of the Working Group on Valvular Heart Diseases, the Working Group on Cardiac Surgery, and the Association of Cardiovascular Interventions of the Polish Cardiac Society. Kardiol Pol. 2020; 78(5): 498-507, doi: 10.33963/KP.15358, indexed in Pubmed: 32415767.

31. Siminiak $T$, Wu JC, Haude $M$, et al. Treatment of functional mitral regurgitation by percutaneous annuloplasty: results of the TITAN Trial. Eur J Heart Fail. 2012; 14(8): 931-938, doi: 10.1093/eurjhf/hfs076, indexed in Pubmed: 22613584

32. Witte KK, Lipiecki J, Siminiak T, et al. The REDUCE FMR trial: a randomized sham-controlled study of percutaneous mitral annuloplasty in functional mitral regurgitation. JACC Heart Fail. 2019; 7(11): 945-955, doi: 10.1016/j. jchf.2019.06.011, indexed in Pubmed: 31521683.

33. Messika-Zeitoun D, Nickenig G, Latib A, et al. Transcatheter mitral valve repair for functional mitral regurgitation using the Cardioband system: 1 year outcomes. Eur Heart J. 2019; 40(5): 466-472, doi: 10.1093/eurheartj/ehy424, indexed in Pubmed: 30124798.

34. Colli A, Manzan E, Aidietis A, et al. An early European experience with transapical off-pump mitral valve repair with NeoChord implantation. Eur J Cardiothorac Surg. 2018; 54(3): 460-466, doi: 10.1093/ejcts/ezy064, indexed in Pubmed: 29514183.

35. Gammie JS, Bartus K, Gackowski A, et al. Beating-Heart mitral valve repair using a novel ePTFE cordal implantation device: a prospective trial. J Am Coll Cardiol. 2018; 71(1): 25-36, doi: 10.1016/j.jacc.2017.10.062, indexed in Pubmed: 29102688. 